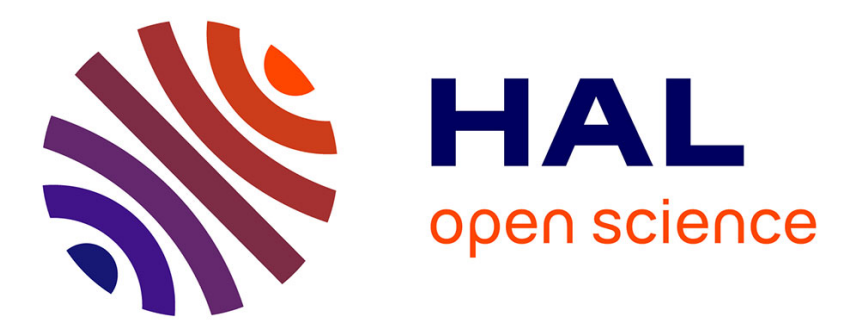

\title{
Short- and Long-Wavelength TIDs Generated by the Great American Eclipse of 21 August 2017
}

\author{
J. Eisenbeis, G. Occhipinti, E. Astafyeva, Lucie Rolland
}

\section{To cite this version:}

J. Eisenbeis, G. Occhipinti, E. Astafyeva, Lucie Rolland. Short- and Long-Wavelength TIDs Generated by the Great American Eclipse of 21 August 2017. Journal of Geophysical Research Space Physics, 2019, 124 (11), pp.9486-9493. 10.1029/2019JA026919 . hal-02553710

\author{
HAL Id: hal-02553710 \\ https://hal.science/hal-02553710
}

Submitted on 31 Mar 2021

HAL is a multi-disciplinary open access archive for the deposit and dissemination of scientific research documents, whether they are published or not. The documents may come from teaching and research institutions in France or abroad, or from public or private research centers.
L'archive ouverte pluridisciplinaire HAL, est destinée au dépôt et à la diffusion de documents scientifiques de niveau recherche, publiés ou non, émanant des établissements d'enseignement et de recherche français ou étrangers, des laboratoires publics ou privés. 


\section{JGR Space Physics}

\section{RESEARCH ARTICLE 10.1029/2019JA026919 \\ Short- and Long-Wavelength TIDs Generated by the Great American Eclipse of 21 August 2017}

Key Points:

- GNSS observations of solar eclipse show differential total electron content (dTEC) depletions reaching $-0.4 \mathrm{TECU}$ in the path of totality

- We observe longer wavelength TIDs that are related to the TEC depletion due to the Moon's shadow, as well as shorter wavelength TIDs

- The omega-k analysis using 3-D FFT allows to highlight the characteristics of the main and more energetic generated TIDs and bow waves.

Supporting Information:

- Supporting Information S1

- Figure S1

- Figure S2

- Movie S1

- Movie S2

- Movie S3

Correspondence to:

J. Eisenbeis,

eisenbeis@ipgp.fr

Citation:

Eisenbeis, J., Occhipinti, G., Astafyeva, E., \& Rolland, L. (2019). Short- and long-wavelength TIDs generated by the Great American Eclipse of 21 August 2017. Journal of Geophysical Research: Space Physics, 124, 9486-9493. https:// doi.org/10.1029/2019JA026919

Received 4 MAY 2019

Accepted 25 SEP 2019

Accepted article online 11 OCT 2019

Published online 16 NOV 2019

(C)2019. American Geophysical Union. All Rights Reserved.

\author{
J. Eisenbeis ${ }^{1}$ iD, G. Occhipinti ${ }^{1,2}$ iD, E. Astafyeva ${ }^{1}$ iD, and L. Rolland ${ }^{3}$ \\ ${ }^{1}$ Institut de Physique du Globe de Paris, Université de Paris, CNRS, Paris, France, ${ }^{2}$ Institut Universitaire de France, Paris, \\ France, ${ }^{3}$ Observatoire de la Côte d'Azur, Université Côte d'Azur, CNRS, IRD, Géoazur, Sophia-Antipolis, France
}

\begin{abstract}
On the 21 August 2017 the eclipse shadow drastically changed the state of the ionosphere over the United States. This effect on the ionosphere is visible in the total electron content measured by Global Navigation Satellite Systems (GNSS). The shadow moved with the supersonic speed of $\sim 1,000 \mathrm{~m} / \mathrm{s}$ over Oregon to $\sim 650 \mathrm{~m} / \mathrm{s}$ over South Carolina. In order to exhaustively explore the ionospheric signature of the eclipse, we use data of total electron content from $~ 3,000$ GNSS stations seeing multiple Global Positioning System (GPS) and Global Navigation Satellite System (GLONASS) satellites to visualize the phenomena. This tremendous dataset allows high-resolution characterization of the frequency content and wavelengths - using an omega-k analysis based on 3-D fast Fourier transform-of the eclipse signature in the ionosphere in order to fully identify traveling ionospheric disturbances (TIDs). We confirm the generation of TIDs associated with the eclipse including TIDs interpreted as bow waves in previous studies. Additionally, we reveal, for the first time, short (50-100 km) and long (500-600 km) wavelength TIDs with periods between 30 and $65 \mathrm{~min}$. The sources of the revealed short wavelength TIDs are co-located with the regions of stronger gradient of the EUV related to sunspots. Our work confirms and describes physical properties of the waves observable in the ionosphere during the Great American Eclipse.

Plain Language Summary A total solar eclipse occurred on 21 August 2017 over the United States with the eclipse shadow reaching supersonic speed. We calculate the differential total electron content to visualize the evolution of the phenomena with incredible high spatial and temporal resolution. Traveling ionospheric disturbances associated with the eclipse with a period of up to $1 \mathrm{hr}$ and wavelengths of 40 to $600 \mathrm{~km}$ including bow waves with a period of $\sim 25$ min and wavelength of $\sim 300 \mathrm{~km}$ are identified using an omega-k analysis based on 3-D fast Fourier transform. The additionally highlighted shortest (50-100 km) and longest (500-600 km) wavelength traveling ionospheric disturbances have not been observed before. The shortest waves seem to be related to sunspots.
\end{abstract}

\section{Introduction}

During the total solar eclipse of 21 August 2017 the Moon's shadow travels with supersonic speed $(\sim 1,000-650 \mathrm{~m} / \mathrm{s})$ from the west to the east coast of the continental United States. It takes about $95 \mathrm{~min}$ for the shadow to cross the United States. Called the Great American Eclipse nowadays, the first total eclipse visible in North America in the XXI century, it also traverses a dense network of $~ 3,000$ Global Navigation Satellite Systems (GNSS) stations giving, for the first time, the unique opportunity to analyze and deeply explore the effect of the Moon's shadow on the ionosphere with high spatial resolution. The Moon's shadow crosses the North American continent from 10:00 local time (LT) in Oregon to 15:00 LT in South Carolina. Therefore, the largest eclipse effects would be expected over the central United States between local noon and 13:00 LT, when the solar radiation is highest. The radical changing of the solar radiation due to the Moon's shadow produced a quick electron density decrease in the ionosphere, clearly visible in the total electron content (TEC) measured by GNSS stations. Indeed, the TEC represents the electron density integrated along a ray-path between a GNSS station and a satellite. The magnitude of the TEC depletion produced by the Moon's shadow was already studied in the past and was found to depend on the latitude (Le et al., 2009). Other studies confirmed this effect: for total solar eclipses the TEC depletion can reach 30-40\% at midlatitudes with a delay of 5-20 min after the passing of the Moon's shadow (Jakowski et al., 2008); (Ding et al., 2010), 10-30\% at high latitudes (Afraimovich et al., 1998); (Momani et al., 2010); (Hoque et al., 2016), whereas for the equatorial latitudes it can reach more than $40 \%$ due to the increased 
ionization within the equatorial anomaly (Tsai \& Liu, 1999). The effect related to the latitude mainly depends on the elevation angle of the Sun and therefore higher ionization at low latitudes, which increases the background TEC. This amplifies the effect of the solar eclipse.

The TEC depletion due to the Moon's shadow and the related change of the solar flux is not the only effect that is expected to occur during the eclipse. The supersonic speed of the shadow of the Moon is predicted to excite lower atmospheric acoustic and gravity waves that can reach the ionosphere and generate various traveling ionospheric disturbances (TIDs), some of them explained as bow waves (Chimonas, 1970); (Chimonas \& Hines, 1971) that are preceding and trailing the solar eclipse and are produced by the reduction of heating sources (e.g., ozone at $45 \mathrm{~km}$ or extreme ultraviolet heating above $100 \mathrm{~km}$ ). TIDs generated by the eclipse have been found to have a wide range of wave periods, ranging from 4 min ((Sauli et al., 2006)), 60 min ((Jakowski et al., 2008)), and to $87 \mathrm{~min}$ (Liu et al., 1998). Additionally, TIDs with a period of 20-40 min have been interpreted as bow waves (Ivanova et al., 1998),

First results for the 21 August 2017 solar eclipse confirmed the expectations by detecting a TEC depletion due to the Moon's shadow as well as the consequent generation of bow waves induced by the supersonic speed of its moving, and other TIDs were clearly visible in the TEC thanks to the high spatial resolution of the dense GNSS network over the entire United States (Zhang et al., 2017); (Coster et al., 2017); (Cherniak \& Zakharenkova, 2018); (Sun et al., 2018);. Those first studies mainly used keograms to obtain information about the TIDs. (Pradipta et al., 2018) confirmed those first observations and the predictions by (Huba \& Drob, 2017) additionally using ionosonde data. (Nayak \& Yigit, 2018) performed a 2-D fast Fourier transform (FFT)to analyze the frequency content of the TIDs at selected stations. Their results showed TIDs with a dominant peak at about 20-30 min and broadly extended to 20-90 min periods, without details about the wavelength of the observed TIDs. A simulation of bow waves during the eclipse (Lin et al., 2018) reproduced TIDs with a period of 20-30 min and therefore similar characteristics to the bow waves observed by (Zhang et al., 2017). They also fall within the range of the dominant frequency peak of the TIDs observed by (Nayak \& Yigit, 2018). The modeled thermospheric/ionospheric bow waves by (Lin et al., 2018) and (Lei et al., 2018) are evanescent in situ generated bow waves (Ridley et al., 1984) and are not a wavefield generated at stratospheric heights as predicted by (Chimonas, 1970). (Harding et al., 2018) present observational evidence for those thermospheric bow waves using direct measurements of a neutral wind disturbance on the nightside. A simulation of the global upper atmospheric responses to the eclipse by (Dang et al., 2018) shows that the effect of the eclipse is not just regional but global and that large-scale atmospheric disturbances were triggered by the eclipse. Thermospheric winds lead to a large TEC enhancement over South America. (Aryal et al., 2019) observe wave-like perturbations in red and green line brightness from ground-based optical measurements. Although the ionosphere-thermosphere system could still be perturbed by the solar eclipse they link LSTIDs generated during the night, with a dominant period of about $1.5 \mathrm{hr}$ and a long wavelength of more than $1,000 \mathrm{~km}$, to an increased geomagnetic activity.

The debate about the physical origin of the perturbations and waves observed during the eclipse in the ionosphere is still open. For instance, (Mrak, Semeter, Drob, et al., 2018) name the modulation of the extreme ultraviolet (EUV) related to high-energy sunspots as the origin of the TIDs instead of the generation of bow waves. More recent work of (Mrak, Semeter, Nishimura, et al., 2018) claim that thunderstorm initiated gravity waves are also a source for observed TIDs before as well as during the eclipse, in addition to the EUV.

Here we present-for the first time-a complete omega-k analysis, based on a 3-D FFT, of the TEC variations observed by GNSS during the solar eclipse over the entire United States to extensively highlight the main periods, wavelengths and speeds of the observed waves and to separate the signatures of different observed waves.

\section{Data and Methodology}

In order to visualize the TEC perturbations induced by the solar flux variations related to the Great American Eclipse, we used an extensive database consisting of 3,000 GNSS stations and capturing signals from Global Positioning System (GPS) and Global Navigation Satellite System (GLONASS) satellites. Using both GPS and GLONASS increases the number of available data by a factor of 1.5-2 compared to the use of GPS only. The used ground-based stations continuously record data with a sampling rate of 30s. We only use 

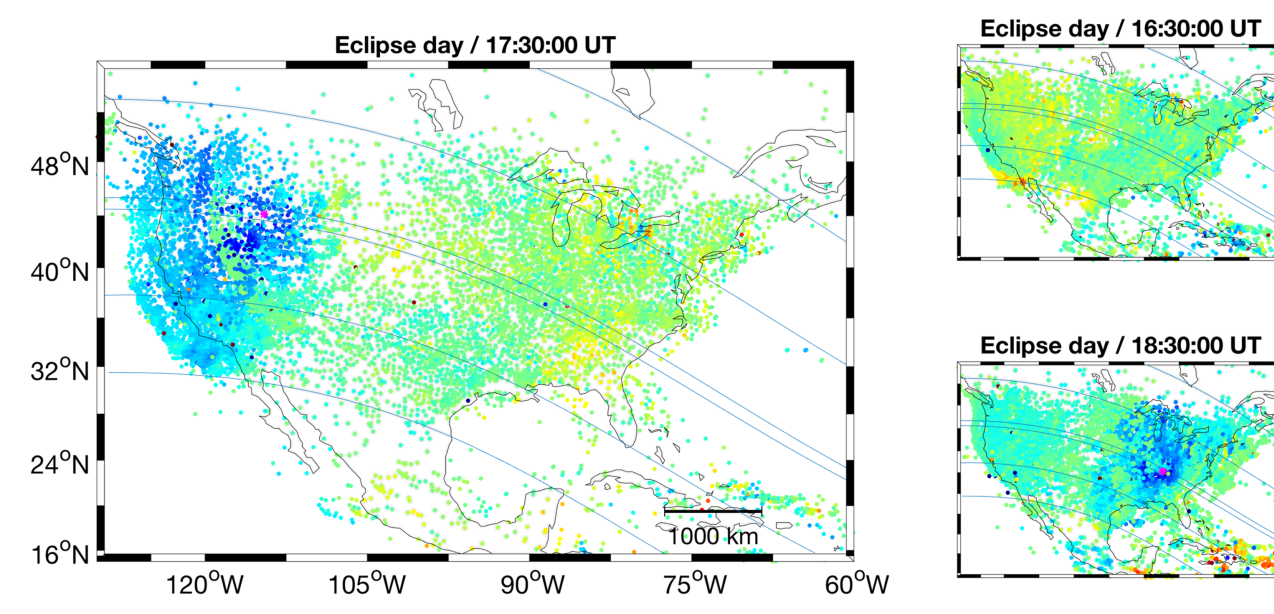

TECU
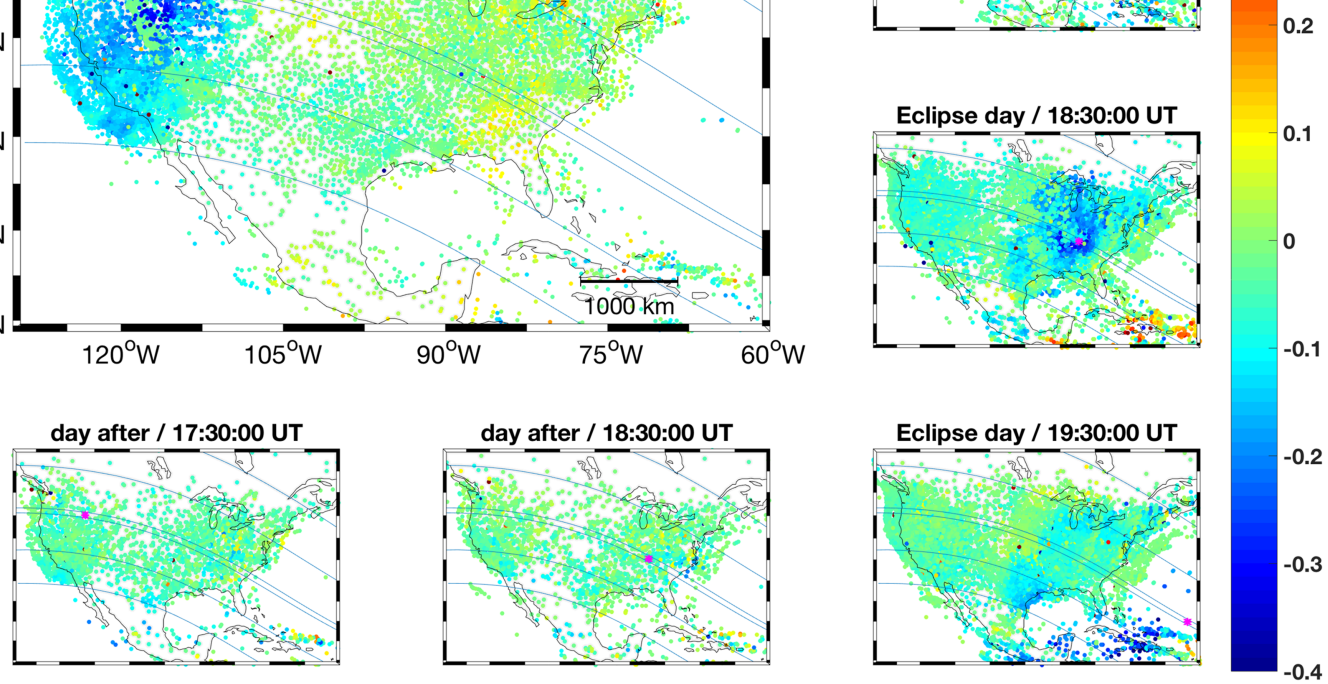

Figure 1. Total electron content (TEC) maps for the eclipse day (top left and right side) and for 22 August, the day after the eclipse (bottom, left and center). Shown are differential vertical TEC (vTEC) values for four moments of time during the totality passing over the United States (16:30 UT, 17:30 UT, 18:30 UT, and 19:30 UT). The location of each observation is the piercing point at $300 \mathrm{~km}$ of altitude. The blue lines are the northern and southern boundary of the totality, $80 \%$ and $60 \%$ of obscuration starting from the magenta star that shows the location of the center of totality. The entire movies are available as Movies S1 and S2.

data of satellite-station pairs with an elevation angle of more than $15^{\circ}$. As mentioned above, the TEC is the integral of the electron density along the line-of-sight between the ground-based GNSS receiver and the satellite. The slant TEC (sTEC) for each satellite-receiver pair is converted to vertical TEC (vTEC) for the height of the maximum ionization of the ionosphere, so it represents the vTEC directly above the receiver. For the conversion of slant TEC to vertical TEC we use the following mapping function (Dautermann \& Calais, 2008):

$$
\mathrm{vTEC}=\operatorname{sTEC}^{*} \sqrt{1-\left(\frac{\cos (\theta) R_{E}}{R_{E}+h_{I}}\right)^{2}}
$$

where $\theta$ is the satellite elevation angle, $R_{E}$ is the mean radius of the Earth, and $h_{I}$ the height of maximum ionization in the ionosphere (here $300 \mathrm{~km}$ ).

In order to compute the differential vTEC to use for the omega-k-analysis, we define the unperturbed background as the polynomial fit of 10th degree of our data. According to (Mrak, Semeter, Drob, et al., 2018) the polynomial fit of 10th degree creates artificial maxima and therefore they used a different approach splitting the data and using two polynomials of varying degree. By filtering the raw data using a butterworth bandpass filter from 0.2 to $2 \mathrm{MHz}$ we can show that those maxima are also present in the filtered data and therefore are not artificial (Figure S2 in the supporting information). The differential vTEC for each receiver-satellite couple is computed separately. The differential vTEC -shown in detail in the supplementary materials (Movies S1 and S2 in the supporting information) and resumed in Figure 1, was computed using only a running mean filter to highlight the TEC depletion due to the eclipse. Further analysis of the eclipse induced phenomena by omega-k analysis is done using the polynomial fit as the only filtering as this provides the best signal to noise ratio. 
Our omega-k analysis consists of detecting plane wave structures of the form $A_{0} e^{-i(\boldsymbol{k} \cdot \boldsymbol{r}+\omega \boldsymbol{t})}$ in the spacetime domain of the differential vTEC dataset, where omega $\omega$ represents the wave's angular frequency which equals to $2 \pi / T$, where $T$ is the period of the wave, and $\boldsymbol{k}$ is the wave vector, which is the vector with a length equal to the wavenumber $k$ in the direction perpendicular to the wavefronts. This means $|\boldsymbol{k}|=k=2 \pi / \lambda$. The direction of the wave vector $\boldsymbol{k}$ is ordinarily the direction at which the plane wave is traveling. This signal representation is adequate to detect the travelling waves signature by both measuring their characteristic temporal scale as well as their physical extent in the considered region. For the computation of the omega-k analysis we use the FFT algorithm (Bracewell \& Bracewell, 2000).

For the omega-k analysis we use differential vTEC computed with a polynomial fit as described above. This gives us a better signal to noise ratio performing the Fourier transform compared to the use of the running mean or bandpass filter and therefore enables us to detect and differentiate the TIDs. Note that no further filtering is performed, theoretically allowing the detection of low-frequency waves with periods larger than $1 \mathrm{hr}$. The discrete values for the angular frequency $\omega$ depend on the length of the total duration $T_{\text {length }}$ of our differential vTEC dataset and the sampling rate $d t$, where $\omega$ ranges from $-\pi / d t$ to $\pi / d t$ in steps of $2 \pi / T_{\text {length }}$. The discrete values of the $k$-vector $\mathbf{k}\left(k_{x}, k_{y}\right)$ are described as follow: $k_{x}$ depends on the size dlx of the cell in longitude and the size $L_{x}$ of the whole grid's dimension in longitude $(\sim 4,600 \mathrm{~km})$; consequently, $k_{x}$ ranges from $-\pi / \mathrm{dlx}$ to $\pi / \mathrm{dlx}$ in steps of $2 \pi / L_{x}$. Following the same description, $\mathrm{k}_{\mathrm{y}}$ depends on dly and Ly, respectively the cell size and the whole grid's dimension in latitude $(\sim 2,200 \mathrm{~km})$. The $\omega$ and $\mathbf{k}\left(k_{x}, k_{y}\right)$ are related to period $T=2 \pi / \omega$ and wavelengths $\lambda\left(\lambda_{x}, \lambda_{y}\right)=\left(2 \pi / k_{x}, 2 \pi / k_{y}\right)$ and expressed in minutes and kilometer, respectively, to simplify the description of the observed TIDs.

In order to perform the omega-k analysis of the ionospheric signature of the eclipse, the differential vTEC is averaged and binned into a grid laid over the United States from $30^{\circ}$ to $50^{\circ}$ in latitude and $235-290^{\circ}$ in longitude with cells of $0.28^{\circ} \times 0.39^{\circ}$ in latitude/longitude, respectively. No interpolation is applied, so that cells without data are marked as blank. The blank cells are set to zero. They correspond to around $11 \%$ of our grid cells. As we use differential TEC for the TEC maps, setting blank cells to zero implies that there is no variation of the TEC in that area. The maximum number of station satellite pairs binned in one pixel varies from 60 to 100 for each epoch, whereas the average number of dTEC measurements per cell is around 35. The 3-D FFT is computed by doing a 1-D FFT along each dimension of the three dimensional array. The TEC maps were created each 30s for a period of $4 \mathrm{hr}$ starting at 16:18 UTC, which is $1 \mathrm{hr}$ before the center of the eclipse first enters Oregon. The resulting 3-D box of 2-D TEC maps per 30 seconds over $4 \mathrm{hr}$ is used as input for a 3-D fast Fourier transform. This tremendous dataset allows a deep analysis of the frequency content and wavelength of the eclipse signature in the ionosphere in order to fully identify the waves associated with the eclipse phenomena.

To highlight the TIDs detected in the omega-k space, we perform an inverse 3-D FFT to show the propagation of the TID in time domain. The inverse 3-D FFT is performed for only the part of the cube identified as contributing to the TID and all other cells are set to zero.

\section{Results}

Figure 1 shows the differential vTEC map for four different time steps during the eclipse, from 16:30 UT before the Moon's shadow appears over Oregon until 19:30 UT when the Moon's shadow moved past North America. For better comparison with the day before and after the eclipse we compute absolute sTEC. To correct for the satellite bias we use the values from the CODE IONEX files of the University of Bern. The receiver bias is removed using the minimum-scalloping method (Rideout \& Coster, 2006). We compare the absolute sTEC single station-satellite of the eclipse day with the day before and the day after the eclipse, in order to highlight the level of perturbation - reaching around 5 TEC unit (TECU), and corresponding to $25 \%$ of the background TEC, produced by the eclipse (Figure S1). We also show differential vTEC maps in order to highlight higher frequency perturbations and wave propagation (Figure 1). Before the Moon's shadow arrives over Oregon, no ionospheric disturbances are detectable in the differential vTEC map. A strong depletion of $\sim 0.4$ TECU is located in the area corresponding to the totality and it affects large parts of the United States until the $60 \%$ of obscuration. The eclipse TEC depletion is clearly visible in the TEC maps from 17:30 to 19:30 UT. The largest depletion has a time lag of $\sim 10$ min to the center of the total eclipse, showing the time of reaction of the ionosphere to the change of solar flux. The TEC 

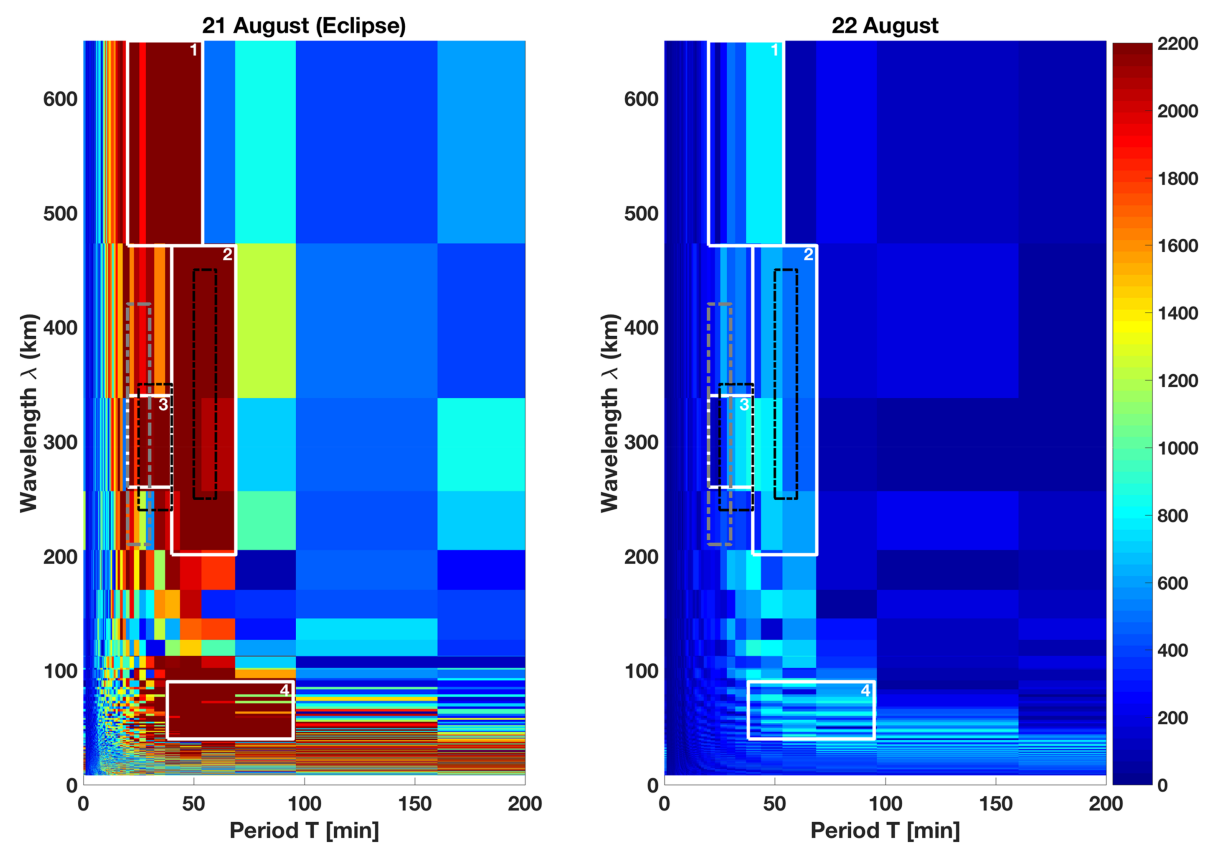

Figure 2. 3-D fast Fourier transform result for the day of the eclipse and the day after. The diagrams show the wavelength $\lambda$ over the period $T$ of the observed waves. Colors show the intensity for specific wavelengths and periods. White boxes numbered one to four highlight the zones of high intensity associated with TIDs following the eclipse. The phase speed range for those boxes is as follows: 145-542 m/s (box 1), 49-196 m/s (box 2), 108-283 m/s (box 3) and 16-39 m/s (box 4). The black dashed boxes correspond to the observations and interpretation of (Zhang et al., 2017): TIDs generated by the TEC depletion by the Moon's shadow (box 2) and by bow waves (box 3). The grey dashed box corresponds to modeled bow waves (Lin et al., 2018). The day after the eclipse is plotted for comparison and to show that the observed TIDs are not daily reoccurring but associated with the solar eclipse.

depletion follows the Moon's shadow during its movement across the United States and the TEC background returned to normal level after the Moon's shadow left North Carolina. Besides the depletion caused by the Moon's shadow other waves are visible, identified as general TIDs and also more specific bow waves preceding and following the main TEC depletion. Bow waves are expected to form if the penumbra moves faster than the local sound speed (Chimonas, 1970), (Chimonas \& Hines, 1971). The sound speed in the ionosphere ranges from $600 \mathrm{~m} / \mathrm{s}$ to $800 \mathrm{~m} / \mathrm{s}$ (Picone et al., 2002). Therefore the speed of the Moon's shadow is supersonic on the west coast $(\sim 1,100 \mathrm{~m} / \mathrm{s})$ but drops down to around sound speed at the east coast $(\sim 650 \mathrm{~m} / \mathrm{s})$ of the United States.

To further investigate the physical characteristics of the TIDs, we perform the omega-k analysis on the differential vTEC maps using the 3-D FFT method on a grid of $0.28^{\circ} \times 0.39^{\circ}$ in cell size in latitude/longitude. This means the size of a pixel is around $33 \mathrm{~km}$ in latitude and ranging from $\sim 29 \mathrm{~km}$ at $50^{\circ} \mathrm{N}$ to $\sim 38 \mathrm{~km}$ at $30^{\circ} \mathrm{N}$ in longitude. As mentioned above, the differential vTEC values were averaged for each cell and no interpolation applied. Cells without data were set to zero. The results of the 3-D FFT show the wavelength over the period (Figure 2) to identify propagating waves. For comparison, the results for the day after the eclipse (22 August) are also shown to highlight that the observed TIDs (on 21 August) are induced by the solar eclipse and are not daily reoccurring events. To support our comparison between the two observed days, we highlight that geomagnetic activity for both days was low, so that the solar eclipse is left as the most likely explanation for the generation of the observed TIDs. In Figure 2 we see four zones with higher intensity, representing TIDs. To define the zones we look for cells with a significant higher intensity as the background and with neighboring cells of the same intensity. We define the boxes trying to avoid including areas of lower intensity. For example, Box 4 could be extended to include a bit bigger wavelengths but not without also including cells of lower intensity. Also, it looks like for small wavelengths and long periods of 100-200 min that there could be a zone of high intensity, but this is regularly intercepted by cells of lower intensity and therefore not considered as TID in this study. Zone 2 shows waves with period of 40-70 min 

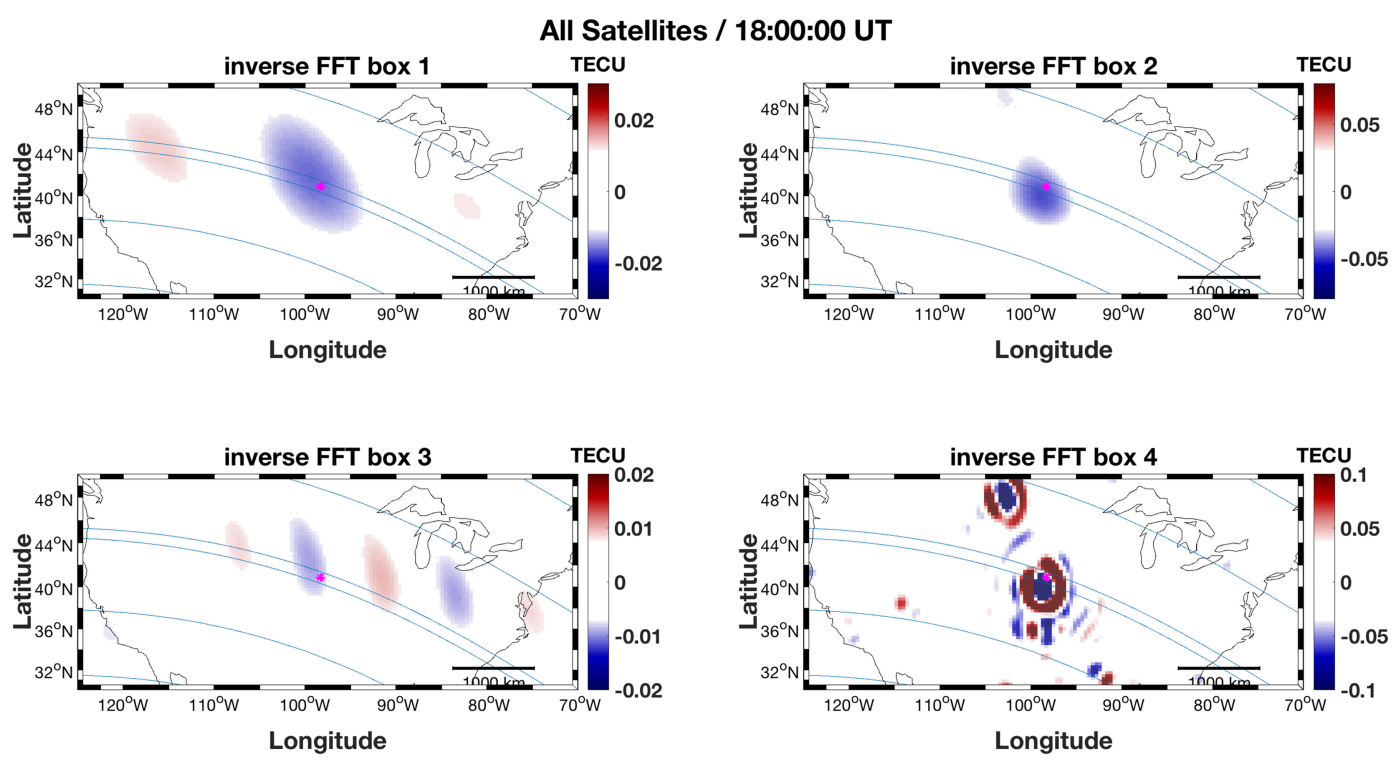

Figure 3. Results for boxes 1-4 from Figure 2 after filtering the data for characteristics of each box in frequency domain and performing an inverse 3-D fast Fourier transform (FFT). Shown is an image for each box at 18:00 UTC to highlight the characteristics of the TIDs. As in Figure 1 the magenta star shows the center of the totality at a specific time and the blue lines represent the northern and southern boundaries of the path of totality and 80/60\% of obscuration respectively.

and wavelength of 200-450 km, which confirms the observations of (Zhang et al., 2017); they speculate that the source of those TIDs is the TEC depletion induced by the solar eclipse. In essence, (Zhang et al., 2017) suggest that the TIDs are directly generated by the plasma density variation in the ionosphere. Zone 3 , related to a period of 25-30 min and wavelength of $300 \mathrm{~km}$, has been interpreted by (Zhang et al., 2017) using keograms-as bow waves induced by the supersonic speed of the shadow. Indeed, zone 3 also corresponds to physical properties of bow waves modeled by (Lin et al., 2018) where the exact eclipse parameters were used to model the generation of bow waves in the low atmosphere, then propagating to the ionosphere. Additionally we found TIDs with period of 40-65 min and wavelength of 50-100 km (zone 4), as well as TIDs with period of 30-60 min and wavelength of 500-600 km (zone 1). The TIDs with physical properties corresponding to zones 1 and 4 were not yet reported in literature. They correspond to long (zone 1) and short (zone 4) wavelength gravity waves that are potentially generated in the low neutral atmosphere and perturb the ionosphere during their upward propagation or are directly generated at ionospheric altitude by the quick plasma density change as speculated by (Zhang et al., 2017). Additionally, the short wavelength gravity waves related to zone 4 present localized sources that around 18:00 UT correspond to the regions of stronger gradient of EUV as suggested by (Mrak, Semeter, Drob, et al., 2018) and support their hypothesis of the role of the modulation of sunspot in the ionospheric signature of the eclipse (Figure 3). Our results support the idea that the localized effect of sunspot generates short wavelength TIDs moving at around 16-39 m/s (zone 4) that coexist with bow waves (zone 3 and 1 ) and other TIDs (zone 2) related to the eclipse.

In order to better visualize the TIDs related to the four boxes, Figure 3 shows the results of an inverse 3-D FFT of each box identified in Figure 2. It shows the propagation of those TIDs in time domain after filtering in the omega-k space for the characteristics seen in Figure 2. As described above, box 2 represents the TEC depletion by the shadow of the Moon itself and it is clearly visible in Figure 3 in the plot to the upper right that the depletion corresponds to the position of the totality with a small time lag. Box 1 shows the same correlation with the totality for a longer wavelength following in the North the shape of the bow waves modeled by (Lin et al., 2018). Same as box 3 it represents bow waves excited during the eclipse but with a longer wavelength than reported for this eclipse before. Box 3 is linked to show the same characteristics as the TIDs described as bow waves by (Zhang et al., 2017) or modeled by (Lin et al., 2018) and as we see in Figure 3 in the bottom left plot it is not only limited to the position of totality but seems to create a wavefield trailing and preceding the totality. This last feature is also present in box 1 . We highlight that boxes 1 and 3 better 
describe the northern extent of the bow waves instead of the v-shape. This effect is the consequence of the larger extent of the bow wave in the North (e.g., Figure 1 at 18:30 UT) coupled together with our methodology that is more sensitive to detecting plane wave structures instead of v-shape structures. Box 4 shows circular structures that seem related to a more static source. Their position does not correspond to thunderstorms occurring during the eclipse ((Mrak, Semeter, Nishimura, et al., 2018)), but around 18:00 UT the position corresponds to that of EUV modulations shown by (Mrak, Semeter, Drob, et al., 2018). (Aryal et al., 2019) show that increased geomagnetic activity after the eclipse generated large scale TIDs with a wavelength of more than $1,000 \mathrm{~km}$ and a dominant period of $1.5 \mathrm{hr}$. As our choice of the grid limits the FFT resolution to a maximum wavelength of $600 \mathrm{~km}$ we cannot see TIDs of the same type during the day, although Box 4 lies within the same period.

(He et al., 2018) show results of a 3-D tomography of the solar eclipse focusing only on the depletion due to the Moon's shadow itself without further investigating other TIDs. Notwithstanding additional efforts are necessary to locate the altitude of TID formation, our work clearly quantifies the physical characteristics (period and wavelength) of the observed TIDs, their location and the propagation pattern. Modeling or 3-D high-resolution reconstruction of the ionosphere (tomography) could help to fully understand whether the generation process occurs in the low altitude neutral atmosphere or in the ionosphere.

\section{Conclusion}

The Great American Eclipse moved with a narrow 160-km-wide Moon's shadow over the entire North America during daytime (10:00-15:00 LT) and along a path from Oregon to North Carolina. Outside of the totality zone, a partial solar eclipse associated with the penumbra covered the majority of the United States. This amazing and unique phenomenon was observed by the wide network of $\sim 3,000$ GNSS stations. They showed for the first time, and with an unprecedently high spatial and time resolution, the response of the ionosphere visualized by TEC measurements all around North America. The TEC signatures of the eclipse show a strong absolute sTEC depletion of the order of $25 \%$ of the normal background estimated using the day before and day after the eclipse. We particularly image the signature of the eclipse over the entire North American subcontinent computing differential vertical TEC maps based on 3,000 stations and 21 GPS or GLONASS satellites. Our differential vertical TEC maps show a depletion of - 0.4 TECU in average following the Moon's shadow with a delay of around $10 \mathrm{~min}$ and broadly extended (transverse to the path of the eclipse) until the area of $60 \%$ of obscuration (blue lines in Figure 1) in North America. Using different data fitting approaches we can show a proof of the existence of a bow wave trailing the eclipse in the TEC maps. We additionally use the high spatial and temporal resolution of our maps to fully analyze the wave propagation visible in the ionosphere as TIDs related to the Great American Eclipse: we perform an omega-k analysis by a 3-D FFT. We confirm the presence of TIDs with period of 40-70 min and wavelength of 200-450 km already observed in the literature using keograms, as well as the presence of TIDs with a period of 25-30 min and the wavelength of $300 \mathrm{~km}$ possibly triggered by the supersonic speed of the Moon's shadow. We also highlight -for the first time in literature- longer wavelength (500-600 km) TIDs with a period of 30-60 min that are related to the TEC depletion due to the Moon's shadow, as well as shorter wavelength (50-100 km) TIDs with a period of 40-65 min. Those last TIDs have sources co-located with the regions of stronger gradient of EUV at a specific time and consequently support the role of the modulation of sunspot in those waves generation. With our work, we wish to push forward the full interpretation of the effect of the Great American Eclipse and generally the fast changing of the ionospheric plasma density related to quick solar flux variation.

fore scientists and the the GNSS networks in North America operational. This work is entirely based on freely available GNSS data from the following web-resources: UNAVCO (www.unavco.org), NOAA (ftp://geodesy.noaa.gov/cors/rinex/), and SOPAC and CSRC GARNER GPS ARCHIVE (ftp://garner.ucsd.edu/ archive/garner/rinex/). This research is supported by Programme National de Télédétection Spatiale (PNTS), grant PNTS-2014-07; by the CNES, grants GISnet\&Back and IonoRadar; and by the Institut Universitaire de France (IUF). This is IPGP contribution 4053.

\section{References}

Afraimovich, E., Palamartchouk, K., Perevalova, N., Chemukov, V., Lukhnev, A., \& Zalutsky, V. (1998). Ionospheric effects of the solar eclipse of March 9, 1997, as deduced from GPS data. Geophysical Research Letters, 25(4), 465-468.

Aryal, S., Geddes, G., Finn, S. C., Mrak, S., Galkin, I., Cnossen, I., et al. (2019). Multispectral and Multi-instrument Observation of TIDs followint the total solar eclipse of 21 August 2017. Journal of Geophysical Research: Space Physics, 124, 3761-3774. https://doi.org/ 10.1029/2018JA026333

Bracewell, R. N., \& Bracewell, R. (2000). The Fourier Transform and Its Applications. University of California: McGraw Hill.

Cherniak, I., \& Zakharenkova, I. (2018). Ionospheric total electron content response to the great American solar eclipse of 21 August 2017. Geophysical Research Letters, 45, 1199-1208. https://doi.org/10.1002/2017GL075989

Chimonas, G. (1970). Internal gravity-wave motions induced in the Earths atmosphere by a solar eclipse. Journal of Geophysical Research, 75(28), 5545-5551. 
Chimonas, G., \& Hines, C. (1971). Atmospheric gravity waves induced by a solar eclipse, 2. Journal of Geophysical Research, 76(28), 7003-7005.

Coster, A., Goncharenko, L., Zhang, S.-R., Erickson, P., Rideout, W., \& Vierinen, J. (2017). GNSS Observations of ionospheric variations during the 21 August 2017 solar eclipse. Geophysical Research Letters, 44, 12,041-12,048. https://doi.org/10.1002/2017GL075774

Dang, T., Lei, J., Wang, W., Zhang, B., Burns, A., Le, H., et al. (2018). Global responses of the coupled thermosphere and ionosphere system to the August 2017 Great American Solar Eclipse. Journal of Geophysical Research: Space Physics, 123, 7040-7050. https://doi.org/ $10.1029 / 2018 \mathrm{JA} 025566$

Dautermann, T., \& Calais, E. (2008). TEC Data Processing Software. Purdue University.

Ding, F., Wan, W., Ning, B., Liu, L., Le, H., Xu, G., \& Yang, M. (2010). GPS TEC response to the 22 July 2009 total solar eclipse in East Asia. Journal of Geophysical Research, 115, A07308. https://doi.org/10.1029/2009JA015113

Harding, B. J., Drob, D. P., Buriti, R. A., \& Makela, J. J. (2018). Nightside detction of a large-scale thermospheric wave generated by a solar eclipse. Geophysical Research Letters, 45, 3366-3373. https://doi.org/10.1002/2018GL077015

He, L., Heki, K., \& Wu, L. (2018). Three-dimensional and trans-hemispheric changes in ionospheric electron density caused by the great solar eclipse in North America on 21 August 2017. Geophysical Research Letters, 45, 933-940.

Hoque, M. M., Wenzel, D., Jakowski, N., Gerzen, T., Berdermann, J., Wilken, V., \& Minkwitz, D. (2016). Ionospheric response over Europe during the solar eclipse of March 20, 2015. Journal of Space Weather and Space Climate, 6, A36.

Huba, J. D., \& Drob, D. (2017). SAMI3 prediction of the impact of the 21 August 2017 total solar eclipse on the ionosphere/plasmasphere system. Geophysical Research Letters, 44, 5928-5935. https://doi.org/10.1002/2017GL073549

Ivanova, V. A., Ryabova, N. V., \& Shumaev, V. V. (1998). Effect of the solar eclipse of 22 July 1990 at mid-latitude path of HF propagation. Journal of Atmospheric and Solar - Terrestrial Physics, 59, 1013-1016.

Jakowski, N., Stankov, S., Wilken, V., Borries, C., Altadill, D., \& Chum, J. (2008). Ionospheric behaviour over Europe during the solar eclipse of 3 October 2005. Journal of Atmospheric and Solar - Terrestrial Physics, 70, 836-853.

Le, H., Liu, L., Yue, X., Wan, W., \& Ning, B. (2009). Latitudinal dependence of the ionospheric response to solar eclipses. Journal of Geophysical Research, 114, A07308. https://doi.org/10.1029/2009JA014072

Lei, J., Dang, T., Wang, W., Burns, A., Zhang, B., \& Le, H. (2018). Long-lasting response of the global thermosphere and ionosphere to the 21 August 2017 solar eclipse. Journal of Geophysical Research: Space Physics, 123, 4309-4316. https://doi.org/10.1029/2018JA025460

Lin, C. Y., Deng, Y., \& Ridley, A. (2018). Atmosperic gravity waves in the ionosphere and thermosphere during the 2017 solar eclipse. Geophysical Research Letters, 45, 5246-5252. https://doi.org/10.1029/2018GL077388

Liu, J. Y., Hsiao, C. C., Tsai, L. C., Liu, C. H., \& Kuo, F. S. (1998). Vertical phase and group velocities of internal gravity waves derived from ionograms during the solar eclipse of 24 October 1995. Journal of Atmospheric and Solar - Terrestrial Physics, 60(1998), $1679-1686$.

Momani, M. A., Yatim, B., \& Mohd Ali, M. A. (2010). Ionospheric and geomagnetic response to the total solar eclipse on 1 August 2008 over Northern Hemisphere. Journal of Geophysical Research, 115, A08321. https://doi.org/10.1029/2009JA014999

Mrak, S., Semeter, J., Drob, D., \& Huba, J. D. (2018). Direct EUV/X-Ray modulation of the ionosphere during the August 2017 total solar eclipse. Geophysical Research Letters, 45, 3820-3828. https://doi.org/10.1029/2017GL076771

Mrak, S., Semeter, J., Nishimura, Y., Hirsch, M., \& Sivadas, N. (2018). Coincidental TID production by tropospheric weather during the August 2017 total solar eclipse. Geophysical Research Letters, 45, 10,903-10,911. https://doi.org/10.1029/2018GL080239

Nayak, C., \& Yigit, E. (2018). GPS-TEC observation of gravity waves generated in the ionosphere during 21 August 2017 total solar eclipse. Journal of Geophysical Research: Space Physics, 123, 725-738. https://doi.org/10.1002/2017JA024845

Picone, J. M., Hedin, A. E., Drob, D. P., \& Alkin, A. C. (2002). NRLMSISE-00 empirical model of the atmosphere: Statistical comparisons and scientific issues. Journal of Geophysical Research, 107(A12), 1468. https://doi.org/10.1029/2002JA009430

Pradipta, R., Yizengaw, E., \& Doherty, P. (2018). Ionospheric density irregularities, turbulence and wave disturbances during the total solar eclipse over North America on 21 August 2017. Geophysical Research Letters, 45, 7909-7917. https://doi.org/10.1029/2018GL079383

Rideout, W., \& Coster, A. (2006). Automated GPS processing for global total electron content data. GPS Solutions, 10, $219-228$.

Ridley, E. C., Dickinson, R. E., Rees, M. H., \& Roble, R. G. (1984). Thermospheric response to the June 11, 1983 solar eclipse. Journal of Geophysical Research, 89(A9), 7583-7588.

Sauli, P., Abry, P., Boska, J., \& Duchayne, L. (2006). Wavelet characterisation of ionospheric acoustic and gravity waves occuring during the solar eclipse of August 11, 1999. Journal of Atmospheric and Solar - Terrestrial Physics, 68(3), 586-598.

Sun, Y.-Y. L.-Y.-H.-Y., Sun, Y.-Y., Liu, J.-Y., Lin, C.-H., Lin, C.-Y., Shen, M.-H., et al. (2018). Ionospheric bow wave induced by the Moon shadow ship over the continent of United States on 21 August 2017. Geophysical Research Letters, 45, 538-544. https://doi.org/10.1002/ 2017GL075926

Tsai, H. F., \& Liu, J. Y. (1999). Ionospheric total electron content response to solar eclipses. Journal of Geophysical Research, 104(A6), 12657-12668. https://doi.org/10.1029/1999JA900001

Zhang, S.-R., Erickson, P. J., Goncharenko, L. P., Coster, A. J., Rideout, W., \& Vierinen, J. (2017). Ionospheric bow waves and perturbations induced by the 21 August 2017 solar eclipse. Geophysical Research Letters, 44, 12,067-12,073. https://doi.org/10.1002/2017GL076054 